\title{
Dabigatran-Induced Massive Spontaneous Hemothorax
}

\author{
Jing Huang ${ }^{1} \cdot$ Wei $\operatorname{Lin}^{1} \cdot \operatorname{Dan} \mathrm{Lv}^{2} \cdot \mathrm{Li} \mathrm{Yu}^{1} \cdot$ Lun $\mathrm{Wu}^{1} \cdot$ Haiying Jin ${ }^{1} \cdot$ \\ Zaichun Deng ${ }^{2} \cdot$ Qunli Ding ${ }^{2}$
}

Published online: 22 September 2017

(C) The Author(s) 2017. This article is an open access publication

\begin{abstract}
Spontaneous hemothorax due to anticoagulant use is extremely rare in clinical practice. Dabigatran is a novel anticoagulant to prevent stroke or thromboembolic episodes in patients with nonvalvular atrial fibrillation. We report on an 83-year-old man who received dabigatran therapy (110 mg twice daily) for 7 months and developed massive spontaneous hemothorax and acute renal failure. The patient was admitted to the hospital with complaint of a dull ache in the chest and dyspnea. Chest computed tomography scan revealed massive pleural effusion in the left hemithorax with atelectasis. Acute renal failure was observed 4 days later after admission. Almost $2500 \mathrm{~mL}$ of blood was repeatedly drained by ultrasound-guided thoracocentesis, followed by a dramatic decrease in serum red blood cell count, hemoglobin and hematocrit. After excluding other possible causes, diagnostic withdrawal was performed for dabigatran, and plasma transfusion was conducted to supply the lost blood volume. A causal relationship was established, because the patient's renal function gradually improved and no further pleural effusion developed after dabigatran was discontinued. This is a rare case report of massive spontaneous hemothorax caused by dabigatran. Therefore, practitioners should be aware of hemothorax as a potential complication of dabigatran therapy.
\end{abstract}

Qunli Ding

ccding2005@163.com

1 Department of Pharmacy, The Affiliated Hospital of Medical College, Ningbo University, No.247, Renmin Road, Jiangbei District, Ningbo 315020, Zhejiang, China

2 Department of Respiratory Medicine, The Affiliated Hospital of Medical College, Ningbo University, No.247, Renmin Road, Jiangbei District, Ningbo 315020, Zhejiang, China

\section{Key Points}

Hemothorax rarely occurs during anticoagulant administration.

Be cautious when using dabigatran in patients with renal insufficiency, in the elderly, or in patients receiving P-glycoprotein inhibitors.

Practitioners should be aware of spontaneous hemothorax as a potential complication of dabigatran therapy.

\section{Introduction}

Hemothorax is a commonly seen problem in clinical practice. It is a condition most commonly attributed to trauma [1]. Spontaneous hemothorax occurs relatively less frequently, and is generally caused by malignancies, anticoagulant therapy, spontaneous pneumothorax, inflammatory process-related blood vessel erosion, hematological abnormalities and pulmonary emboli. It may also result from intrathoracic blood vessel anomalies, pleural adhesion rupture, chest wall exostoses, congenital heart defects and thoracic endometriosis [2-4]. The amount of blood accumulated in the pleural space can vary from minor to massive. Massive hemothorax refers to a blood loss of $>1500 \mathrm{~mL}$ [3].

Anticoagulant-associated spontaneous hemothorax is a well-known yet rarely encountered clinical problem, which may go unnoticed by clinicians and lead to delayed diagnosis. Traditional anticoagulants such as warfarin, heparin 
and enoxaparin have been reported to be responsible for spontaneous hemothorax [2]. Dabigatran is one of the direct oral anticoagulants (DOACs) for nonvalvular atrial fibrillation at risk of stroke or systemic embolism [5]. In the present study, we present a case of massive spontaneous hemothorax during dabigatran therapy.

\section{Case Report}

An 83-year-old man without trauma and surgical history was admitted to our hospital with a dull ache in the chest and dyspnea for 10 days. He had a known diagnosis of hypertension, hyperlipidemia, nonvalvular atrial fibrillation, chronic obstructive pulmonary disease and chronic renal disease, with a past medication history of long-term use of amlodipine besylate ( $5 \mathrm{mg}$ daily), benazepril (10 mg daily), atorvastatin (20 mg daily) and 7 months administration of dabigatran (110 mg twice daily).

Respiratory and heart examination revealed reduced breath sounds and dullness to percussion at the left low base, with irregular heart sounds, but no pathological murmur.

As shown in Fig. 1a, chest computed tomography (CT) scan revealed a massive pleural effusion in the left hemithorax with atelectasis. Vascular structures were normal, and no pulmonary emboli or obvious pericardial effusion was detected. Laboratory results included white blood cell (WBC) count $(10,100 / \mu \mathrm{L})$, neutrophil count $(83.7 \%)$, red blood cell (RBC) count $(3,820,000 / \mu \mathrm{L})$, hemoglobin $(123 \mathrm{~g} / \mathrm{L})$, hematocrit $(0.38)$, blood platelet count $(201,000 / \mu \mathrm{L})$, C-reactive protein $(61.1 \mathrm{mg} / \mathrm{L})$, and procalcitonin $<0.05 \mu \mathrm{g} / \mathrm{L}$. In addition, serum B-type natriuretic peptide (BNP) was $46.8 \mathrm{ng} / \mathrm{L}$, and cardiac troponin I was below $0.01 \mathrm{ng} / \mathrm{mL}$. Coagulation parameters of prothrombin time, international normalized ratio, activated partial thromboplastin time, thromboplastin time, and D-dimer were $15.6,1.35,73.3 \mathrm{~s}$, out of detection, and
$483 \mu \mathrm{g} / \mathrm{L}$, respectively. Serum tumor markers (NSE, CEA and CYFRA-21) were normal.

Acute renal failure was observed within 4 days after admission; the patient's serum creatinine was $159 \mu \mathrm{mol} / \mathrm{L}$ on the first day, $195.1 \mu \mathrm{mol} / \mathrm{L}$ on the second day and $329.6 \mu \mathrm{mol} / \mathrm{L}$ on the fourth day after admission. According to the Cockcroft-Gault formula, the creatinine clearance $(\mathrm{CrCl})$ of the patient quickly decreased from $32.9 \mathrm{~mL} / \mathrm{min}$ (first day) to $15.9 \mathrm{~mL} / \mathrm{min}$.

Ultrasound-guided thoracocentesis was performed. The parameters of the pleural fluid included positive Rivalta test, WBC count of $2800 / \mu \mathrm{L}$ (neutrophils $36 \%$, lymphocytes $56 \%$ and monocyte $8 \%$ ), total protein of $51.8 \mathrm{~g} / \mathrm{L}$, lactate dehydrogenase (LDH) of $377 \mathrm{U} / \mathrm{L}, \mathrm{RBC}$ count of $1,150,000 / \mu \mathrm{L}$, adenosine deaminase (ADA) of $9 \mathrm{U} / \mathrm{L}$ and glucose of $6.57 \mathrm{mmol} / \mathrm{L}$. Data regarding the hematocrit of the effusion were not collected. The thinprep cytologic test (TCT) of the fluid revealed no malignancy. Bacterial smears and cultures of the drainage were negative (Table 1).

The patient's serum RBC count, hemoglobin and hematocrit dramatically dropped from $3,820,000 / \mu \mathrm{L}, 123 \mathrm{~g} / \mathrm{L}$ and 0.38 to $2,530,000 / \mu \mathrm{L}, 80 \mathrm{~g} / \mathrm{L}$ and 0.24 , respectively, after almost $2500 \mathrm{~mL}$ of blood and turbid pleural fluid was aspired from the left hemithorax. Plasma transfusion was conducted to supply the lost blood volume. Diagnostic withdrawal was performed for dabigatran (day 5). The discontinuation of dabigatran led to the resolution of symptoms. Patient renal function was gradually improved after dabigatran was discontinued (Table 2), and no further pleural effusion developed (Fig. 1b). Pleural effusion with moderate visceral (V) and parietal (P) thickening was observed on the left side after conservative treatment (Fig. 1c).

Our patient was later stabilized and dismissed home. Unfortunately, on a visit, he died of sudden cardiac death 6 months after his discharge from our hospital.
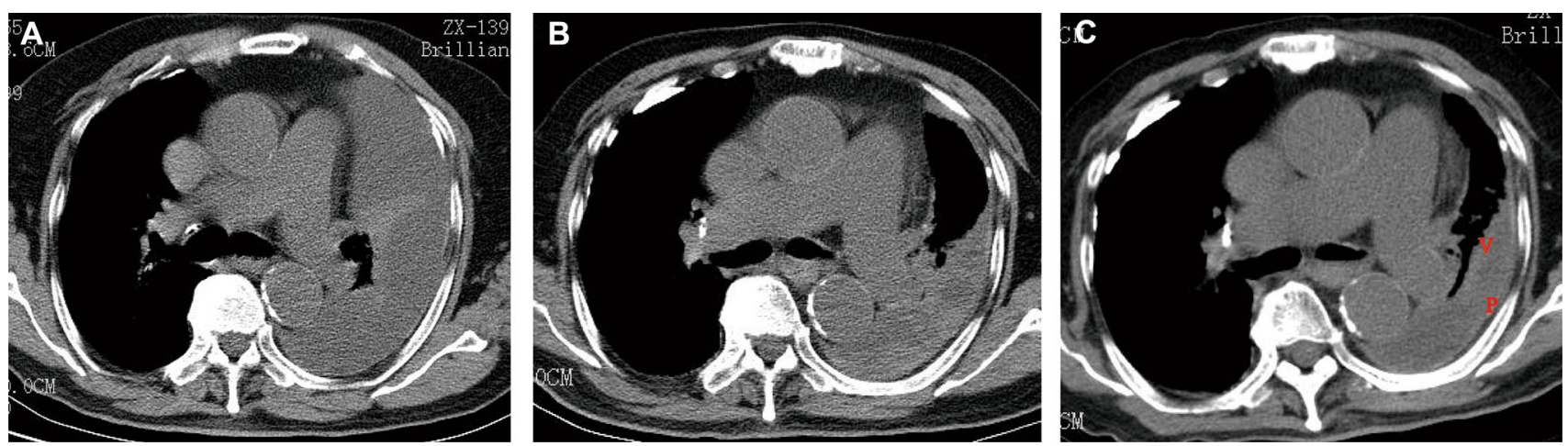

Fig. 1 a Chest CT scan shows a massive pleural effusion on the left side with atelectasis when the patient was admitted; $\mathbf{b}$ chest CT scan after $2500 \mathrm{~mL}$ of pleural fluid was drained and dabigatran was discontinued; $\mathbf{c}$ a chest CT scan shows the left-sided pleural effusion with moderate visceral $(\mathrm{V})$ and parietal $(\mathrm{P})$ thickening. $C T$ computed tomography 
Table 1 Parameters of pleural effusion at different dates after admission

\begin{tabular}{lll}
\hline Parameters & Day 4 & Day 5 \\
\hline Character & Turbid, bloody & Turbid, bloody \\
Rivalta test & Positive & Positive \\
White blood cells $(/ \mu \mathrm{L})$ & 2800 & 2700 \\
Red blood cells $(/ \mu \mathrm{L})$ & $1,150,000$ & $1,140,000$ \\
Neutrophils $(\%)$ & 36 & 34 \\
Lymphocytes $(\%)$ & 56 & 56 \\
Monocyte $(\%)$ & 8 & 7 \\
Total protein $(\mathrm{g} / \mathrm{L})$ & 51.8 & 51.2 \\
Albumin $(\mathrm{g} / \mathrm{L})$ & 30.9 & 30 \\
Lactate deaminase $(\mathrm{U} / \mathrm{L})$ & 377 & 383 \\
Adenosine deaminase $(\mathrm{U} / \mathrm{L})$ & 9 & 9 \\
Glucose (mmol/L) & 6.57 & 5.7 \\
Bacterial culture & Negative & Negative \\
Fungus culture & Negative & Negative \\
\hline
\end{tabular}

\section{Discussion}

Hemothorax is a collection of blood accumulating in the pleural space [3]. There are various etiologies of hemothorax. However, hemothorax secondary to anticoagulant therapy is unexpected. The diagnosis of anticoagulant-induced hemothorax is a challenge for clinicians, which requires high suspicion based on risk factors and differentiation from other causes.

Massive spontaneous hemothorax was considered in our case, since the patient had no trauma or surgery experience. The appearance of the pleural fluid, the LDH, ADA and glucose levels, the different cell counts in the pleural fluid, and other laboratory test results such as microbiologic and cytologic studies, serum BNP level and CT films helped to exclude potential causes such as heart failure, tuberculosis effusion, malignant pleural disease, pneumonia, or pulmonary embolism. Alternatively, this patient had no previous disease of spontaneous pneumothorax and intrathoracic blood vessel anomaly. Bleeding disorder or aortic dissection seemed unlikely. Anticoagulant administration, another important cause of hemothorax, was taken into consideration, since this patient was exposed to a new anticoagulant of dabigatran for 7 months under the condition of nonvalvular atrial fibrillation.

Dabigatran is the first DOAC to prevent stroke or thromboembolic episodes in patients with nonvalvular atrial fibrillation [6]. With well-tolerated, predictable pharmacokinetics, no need for coagulation monitoring and potent anticoagulant effects, dabigatran has been increasingly used in clinical practice. However, a concern remains about its bleeding risk. Bleeding events such as gastrointestinal bleeding, intracranial bleeding and pericardial bleeding, which is sometime serious or even fatal, have been well-documented [7, 8]. However, bleeding manifestation as hemothorax is seldom reported.

Renal insufficiency and P-glycoprotein (P-gp) inhibition are the risk factors for increased dabigatran exposure and the consequent increased bleeding risk. Dabigatran is eliminated primarily by the kidney [9]. The administration of dabigatran in patients with renal impairment leads to the increased dabigatran exposure, prolonged half-life and increased risk for bleeding [5, 10]. The Food and Drug Administration (FDA) approved dabigatran at a dosage of $150 \mathrm{mg}$ twice daily for a $\mathrm{CrCl}$ of $\geq 30 \mathrm{~mL} / \mathrm{min}$, while a dosage of $110 \mathrm{mg}$ twice daily is recommended for patients with a $\mathrm{CrCl}$ of $30-50 \mathrm{~mL} / \mathrm{min}$ in China, Canada and Europe. Furthermore, $75 \mathrm{mg}$ twice daily is recommended for patients with a $\mathrm{CrCl}$ of $15-30 \mathrm{~mL} / \mathrm{min}$. It has been generally recognized that dabigatran should be avoided in patients with a $\mathrm{CrCl}$ of $<15 \mathrm{~mL} / \mathrm{min}$ [11]. However, the elderly are more likely to have reduced renal clearance. The Randomized Evaluation of Long Term Anticoagulation Therapy (RE-LY) study demonstrated a significant treatment-by-age interaction. Compared with warfarin, both dosages of dabigatran (150 and $110 \mathrm{mg}$ twice daily) were associated with less major bleeding risk in patients $<75$ years old. However, in patients $\geq 75$ years old, dabigatran $150 \mathrm{mg}$ twice daily was associated with a higher rate of major bleeding, and dabigatran $110 \mathrm{mg}$ twice daily had a similar risk of bleeding [5]. In addition, dabigatran is a substrate of the efflux transporter P-gp. P-gp inhibitor co-administration is another independent risk factor for increased dabigatran exposure and risk for bleeding [12]. Dosage adjustments are recommended for those with reduced renal clearance, and those who are also taking certain P-gp inhibitors.
Table 2 Renal function indexes at different dates after admission

\begin{tabular}{lcrrrrrr}
\hline Indexes & Day 1 & Day 2 & Day 4 & Day 9 & Day 14 & Day 28 & Day 42 \\
\hline Urea-n $(\mathrm{mmol} / \mathrm{L})$ & 11.66 & 13.16 & 19.14 & 23.78 & 16.04 & 17.22 & 8.53 \\
$\mathrm{Cr}(\mu \mathrm{mol} / \mathrm{L})$ & 159.0 & 195.10 & 329.60 & 263.00 & 207.00 & 145.80 & 96.00 \\
Urea $(\mu \mathrm{mol} / \mathrm{L})$ & 5970 & 651.00 & 774.00 & 854.00 & 699.00 & 751.00 & 391.00 \\
$\mathrm{CrCl}(\mathrm{mL} / \mathrm{min})$ & 32.90 & 26.80 & 15.90 & 19.90 & 25.20 & 35.80 & 54.40 \\
\hline
\end{tabular}

Urea- $n$ blood urea nitrogen, $\mathrm{Cr}$ serum creatinine, $\mathrm{CrCl}$ creatinine clearance 
Thus, older age, renal failure and P-gp inhibitor co-administration were the risk factors that strengthened our suspicion of dabigatran. Dabigatran $110 \mathrm{mg}$ twice daily with no dosage reduction was co-administered with atorvastatin, a P-gp inhibitor. This might have induced the higher dabigatran exposure and increased serum concentration and bleeding risk in the 83-year-old acute renal failure patient. This diagnosis was further supported by remission after discontinuing dabigatran. Utilizing the Naranjo adverse drug reaction probability scale, this adverse reaction is categorized as being probably due to dabigatran [13].

In the literature, hemothorax in the setting of treatment with warfarin [14], heparin [15] and low molecular weight heparin [16] has been well-documented. Most cases usually occur within the first week of the start of anticoagulation therapy, but it may also occur at a later time [2]. However, to the best of our knowledge, only one other report of spontaneous hemothorax has described this to be associated with dabigatran. The patient was a 72-year-old male who developed moderate spontaneous hemothorax in the right lung after receiving $110 \mathrm{mg}$ of dabigatran twice daily for 1.5 years, and this patient was drained of a total of $1400 \mathrm{cc}$ of hemorrhagic fluid [17]. In addition, there was another reported case of a 63-year-old woman who received dabigatran for 8 weeks, causing sanguineous pericardial effusion and left pleural effusion [18].

An additional highlight of our case is that the patient exhibited a rapid reduction in renal clearance. Finally, renal function began to recover (day 9) after dabigatran discontinuation (day 5), which made us believe that the patient's renal function failure was associated with dabigatran treatment. Böhm et al. analyzed changes in renal function during treatment with dabigatran (110 or $150 \mathrm{mg}$ twice daily) in patients enrolled in the RE-LY trial. Patients with atrial fibrillation receiving dabigatran treatment exhibited a decline in renal function in both treatment groups [19]. In addition, the existence of dabigatran-related nephropathy has been considered by some authors [20].

Thus, our patient may be the second case with dabigatran-associated spontaneous hemothorax, but with a higher volume of bleeding. In addition, this is the first case to describe spontaneous hemothorax and acute renal failure in the same patient treated with dabigatran.

\section{Conclusion}

Hemothorax rarely occurs during anticoagulant administration. Once an anticoagulant-induced hemothorax has been identified, drug discontinuation may be all that is needed. Although this occurrence is rare, anticoagulant agents, including DOACs, should always be considered as a potential cause of spontaneous hemothorax.

\section{Compliance with Ethical Standards}

Funding source This work was supported by grants from the Natural Science Foundation of Zhejiang (LY15H010004) and the Natural Science Foundation of Ningbo (2014A610279).

Financial disclosure The authors have no financial relationships relevant to this article to disclose.

Conflict of interest Jing Huang, Wei Lin, Dan Lv, Li Yu, Lun Wu, Haiying Jin, Zaichun Deng and Qunli Ding declare that they have no conflicts of interest relating to the content of this article.

Informed consent A written informed consent was obtained from the patient for the publication of this case report and any accompanying images. A copy of the written consent may be requested for review from the corresponding author.

Open Access This article is distributed under the terms of the Creative Commons Attribution-NonCommercial 4.0 International License (http://creativecommons.org/licenses/by-nc/4.0/), which permits any noncommercial use, distribution, and reproduction in any medium, provided you give appropriate credit to the original author(s) and the source, provide a link to the Creative Commons license, and indicate if changes were made.

\section{References}

1. Boersma WG, Stigt JA, Smit HJM. Treatment of haemothorax. Respir Med. 2010;104:1583-7.

2. Ali HA, Lippmann M, Mundathaje U, Khaleeq G. Spontaneous hemothorax: a comprehensive review. Chest. 2008;134:1056-65.

3. FACS RCMP, FACS DBHM. Chest wall trauma, hemothorax, and pneumothorax. Current surgical therapy, 11th ed. Elsevier Inc.; 2014. pp. 1005-9.

4. Janik M, Straka L, Krajcovic J, Hejna P, Hamzik J, Novomesky F. Non-traumatic and spontaneous hemothorax in the setting of forensic medical examination: a systematic literature survey. Forensic Sci Int. 2014;236:22-9.

5. Waks JW, Zimetbaum PJ. Dabigatran etexilate for thromboembolic prophylaxis in non-valvular atrial fibrillation: the RE-LY study and substudies with commentary. Expert Rev Cardiovasc Ther. 2013;11:1461-71.

6. Enriquez A, Baranchuk A, Redfearn D, Simpson C, Abdollah H, Michael K. Dabigatran for the prevention and treatment of thromboembolic disorders. Expert Rev Cardiovasc Ther Inf Healthc. 2015;13:529-40.

7. Lal Y, Van Heukelom J. Dabigatran: a cause of hematologic emergency. Am J Med Sci. 2013;346:190-3.

8. Kumar R, Smith RE, Henry BL. A review of and recommendations for the management of patients with life-threatening dabigatran-associated hemorrhage: a single-center university hospital experience. J Intensive Care Med. 2015;30:462-72.

9. Blech S, Ebner T, Ludwig-Schwellinger E, Stangier J, Roth W. The metabolism and disposition of the oral direct thrombin inhibitor, dabigatran, in humans. Drug Metab Dispos. 2008;36:386-99.

10. Stangier J, Rathgen K, Stahle H, Mazur D. Influence of renal impairment on the pharmacokinetics and pharmacodynamics of 
oral dabigatran etexilate: an open-label, parallel-group, singlecentre study. Clin Pharmacokinet. 2010;49:259-68.

11. Dobesh PP, Fanikos J. Direct oral anticoagulants for the prevention of stroke in patients with nonvalvular atrial fibrillation: understanding differences and similarities. Drugs. 2015;75:1627-44.

12. Stangier J. Clinical pharmacokinetics and pharmacodynamics of the oral direct thrombin inhibitor dabigatran etexilate. Clin Pharmacokinet. 2008;47:285-95.

13. Naranjo CA, Busto U, Sellers EM, Sandor P, Ruiz I, Roberts EA, et al. A method for estimating the probability of adverse drug reactions. Clin Pharmacol Ther. 1981;30:239-45.

14. Doğan NÖ, Pamukçu Günaydın G, Tekin M, Cevik Y. Nontraumatic massive spontaneous hemothorax with concomitant warfarin use. Case Rep Emerg Med. 2013;2013:1-3.

15. Rostand RA, Feldman RL, Block ER. Massive hemothorax complicating heparin anticoagulation for pulmonary embolus. South Med J. 1977;70:1128-30.
16. Ganguli A, Walker L, FitzGerald RJ, Pirmohamed M. Spontaneous hemothorax following anticoagulation with low-molecularweight heparin. Ann Pharmacother. 2009;43:1528-31.

17. Akgedik R, Günaydin Z, Bektas O, Karagöz A, Öztürk H. Spontaneous hemothorax due to dabigatran use in a patient with atrial fibrillation. Clin Respir J. 2017;11:394-6.

18. Abdallah M, Abdallah T, Abi Rafeh N, Khoueiry G, Abouyassine A, Chalhoub M, et al. A sanguineous pleuro pericardial effusion in a patient recently treated with dabigatran. Heart Lung $\mathrm{J}$ Acute Crit Care. 2015;44:209-11.

19. Böhm M, Ezekowitz MD, Connolly SJ, Eikelboom JW, Hohnloser SH, Reilly PA, et al. Changes in renal function in patients with atrial fibrillation: an analysis from the RE-LY trial. J Am Coll Cardiol. 2015;65:2481-93.

20. Shafi ST, Negrete H, Roy P, Julius CJ, Sarac E. A case of dabigatran-associated acute renal failure. WMJ. 2013;112:173-5 (quiz 6). 